\title{
Dynamic Analysis of Planar 3-RRR Flexible Parallel Robots with Dynamic Stiffening
}

\author{
Qinghua Zhang, ${ }^{1}$ Xuerui Fan, ${ }^{1}$ and Xianmin Zhang $^{2}$ \\ ${ }^{1}$ School of Engineering Technology, Beijing Normal University Zhuhai, No. 18 Jinfeng Road, Tangjiawan, \\ Zhuhai City, Guangdong 519087, China \\ ${ }^{2}$ Guangdong Province Key Laboratory of Precision Equipment and Manufacturing Technology, Wushan Road, \\ Tianhe District, Guangzhou 510641, China
}

Correspondence should be addressed to Qinghua Zhang; qinghuazhang411@163.com

Received 12 November 2013; Accepted 16 January 2014; Published 20 March 2014

Academic Editor: Ahmet S. Yigit

Copyright (C) 2014 Qinghua Zhang et al. This is an open access article distributed under the Creative Commons Attribution License, which permits unrestricted use, distribution, and reproduction in any medium, provided the original work is properly cited.

In consideration of the second-order coupling quantity of the axial displacement caused by the transverse displacement of flexible beam, the first-order approximation coupling model of planar 3-RRR flexible parallel robots is presented, in which the rigid body motion constraints, elastic deformation motion constraints, and dynamic constraints of the moving platform are considered. Based on the different speed of the moving platform, numerical simulation results using the conventional zero-order approximation coupling model and the proposed firstorder approximation coupling model show that the effect of "dynamic stiffening" term on dynamic characteristics of the system is insignificant and can be neglected, and the zero-order approximation coupling model is enough precisely for catching essentially dynamic characteristics of the system. Then, the commercial software ANSYS 13.0 is used to confirm the validity of the zero-order approximation coupling model.

\section{Introduction}

In recent several decades, many researchers have paid more attention to the light flexible robots with high-speed, highacceleration, and high-precision which are widely used in the assembly industry, the aerospace industry and the precision machining, and the measurement field. Essentially, the flexible parallel robots mechanism belongs to flexible multibody system. Dynamic modeling of flexible multibody system is a challenging task, in which not only rigid-flexible coupling effect must be studied but also elastic deformation coupling must be analyzed carefully.

At present, dynamic modeling and control of the flexible multibody system have received considerable attention as seen in survey papers [1-4]. Unfortunately, most of published works in this area addressed the manipulators with one flexible link. Comparing with single-link flexible manipulator, two-link flexible manipulator, or four-bar linkage flexible mechanism $[5,6]$, the research works on the flexible parallel robots are rather few.
Recently, few works have been done on dynamic modeling and control of complex mechanisms. Lee and Geng [7] developed a dynamic model of a flexible Stewart platform using Lagrange equations. Wang et al. [8-10] studied dynamic modeling and control of planar 3-Prismatic-jointRevolute-joint-and-Revolute-joint (3-PRR) parallel robots. Zhang et al. [11, 12] studied dynamic modeling method and dynamic characteristics of planar 3-RRR flexible parallel robots. Q. H. Zhang and X. M. Zhang [13] also studied dynamic performance of planar 3-RRR flexible parallel robots under uniform temperature change.

For a simple flexible hub-beam system, when the system operates at a high speed, the conventional hybrid coordinate model, namely, zero-order approximation coupling model (ZOAC model) that assumes axial and transverse displacement of a point on the neutral axis of a beam are uncoupled is an invalid modeling method [14]. Kane et al. [15] found that when the nominal motion of rigidflexible rotating beam system is very large, the deformation of the flexible system deformation exists significantly warp by 
the conventional dynamic model and experimental method, and he thought the premature linearization would lead to lack of "dynamic stiffening" term and firstly proposed "dynamic stiffening" phenomenon. Since then, many scholars start to study the so-called "dynamic stiffening" terms of rigid-flexible coupling system and different methods are used to capture it [14, 16-18]. Mayo et al. [16] reviewed different formulations to account for the geometric stiffening or dynamic stiffening effect arising from deflections large enough to cause significant changes in the configuration of the system. Lugrís et al. [17] used two different methods to calculate dynamic stiffening of the flexible system: one method is from the deformation energy and the other is from foreshortening. Zhang et al. [18] indicated that when the rotational frequency of slender beams meets or exceeds the fundamental frequency, the elastic deformation of flexible beam that is obtained by the conventional ZOAC model tends to diverge. Yu and Hong [19] summarized several methodologies for analyzing dynamic stiffening of flexible system. In consideration of structural geometric nonlinear effects, Wu and Haug [20] established a substructure synthesis formulation of flexible multibody system. Yang [21] studies dynamic model of rigid-flexible system from both theory and experiment. In consideration of dynamic stiffening effect, Liu and $\mathrm{Lu}$ [22] established the rigid-flexible coupling dynamic equations of three-dimensional hub-beams system using velocity variational principle and finite element method.

Since dynamic stiffening phenomenon was proposed by Kane et al., many relevant literatures were published $[14,16-$ 22 ], but they mainly focus on the flexible rotation beam system. To my knowledge, dynamic stiffening phenomenon of flexible parallel robots has never been studied. Because dynamic stiffening term was not neglected in the high-speed flexible rotation beam system, we do not know that how dynamic stiffening of flexible parallel robots influences its owe dynamic characteristics. So, it is important for us to study dynamic stiffening of flexible parallel robots.

The remainder of the paper is organized as follows. Section 2 introduces dynamic modeling of planar 3-RRR flexible parallel robots with dynamic stiffening. In consideration of the second-order coupling quantity of the axial displacement caused by the transverse displacement of flexible beam, based on the finite element method (FEM) and the Lagrange equation, the first-order approximation coupling model (FOAC model) of planar 3-RRR flexible parallel robots is presented. Section 3 discusses the constraint equations of planar 3-RRR flexible parallel robots system which include rigid body motion constraints, elastic deformation motion constraints, and dynamic constraints of the moving platform. In Section 4, numerical results are presented based on the ZOAC model and FOAC model. One finds that the influence of dynamic stiffening of planar 3-RRR flexible parallel robots on dynamic characteristics can be neglected, and the ZOAC model can effectively reflects dynamic characteristics of the system (Figure 7). The commercial ANSYS 13.0 software is used to confirm the validity of the ZOAC model. Finally, the conclusions are given in Section 5.

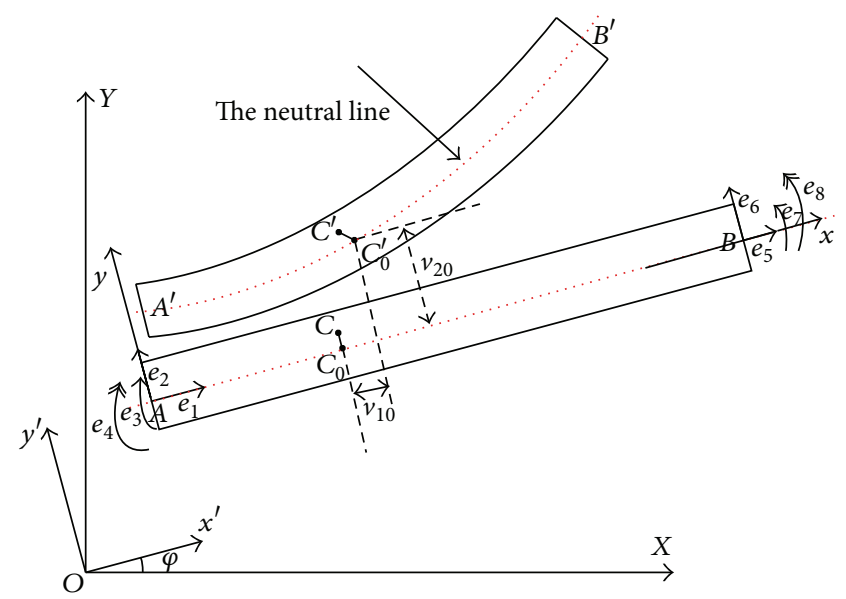

FIGURE 1: Beam element deformation.

\section{Dynamic Modeling of Planar 3-RRR Flexible Parallel Robots with Dynamic Stiffening}

The flexible link can be modeled by connecting a series of beam elements. Figure 1 shows a beam element before and after deformation. The $O-X Y$ is the global fixed frame and the $A-x y$ is the local moving frame with $A x$ axis coincident with the neutral line of the beam element. Its original point $A$ is located at one node of the beam element before deformation. $B$ is another node of the beam element. The $O-x^{\prime} y^{\prime}$ system is an intermediate coordinate frame whose origin is rigidly attached to the origin of the $O-X Y$ and whose axes are parallel to the axes of the local moving frame $A-x y . \varphi$ is the angle between the global fixed frame $O-X Y$ and the intermediate coordinate frame $O-x^{\prime} y^{\prime}$. Considering the general point $C$ in the beam element, let point $C_{0}$ be the corresponding point on the neutral line. Points $C^{\prime}$ and $C_{0}^{\prime}$ are their positions after deformation, respectively. The elastic deformation of the point $C_{0}$ in the $A-x y$ is given by

$$
\mathbf{v}_{0}(x, t)=\left[\begin{array}{l}
v_{10} \\
v_{20}
\end{array}\right]=\overline{\mathbf{N}}(x) \mathbf{e}_{f}
$$

where $\mathbf{e}_{f}=\left[\begin{array}{llllllll}e_{1} & e_{2} & e_{3} & e_{4} & e_{5} & e_{6} & e_{7} & e_{8}\end{array}\right]^{T}$ is the nodal displacement vector, in which $e_{1}$ and $e_{5}$ are the axial displacements of two nodes $A$ and $B$, respectively; $e_{2}$ and $e_{6}$ are the transverse displacements; $e_{3}$ and $e_{7}$ are elastic rotational angles; $e_{4}$ and $e_{8}$ are section curvatures; and the superscript $T$ indicates matrix transpose. $\overline{\mathbf{N}}(x)$ is the shape function matrix. Assuming that the axial displacement $\mathbf{v}_{10}$ of point $C_{0}$ is a linear function, the transverse displacement $\mathbf{v}_{20}$ is a fifthorder hermit function, yielding

$$
\overline{\mathbf{N}}=\left[\begin{array}{l}
\overline{\mathbf{N}}_{1}(x) \\
\overline{\mathbf{N}}_{2}(x)
\end{array}\right]=\left[\begin{array}{cccccccc}
f_{1} & 0 & 0 & 0 & f_{5} & 0 & 0 & 0 \\
0 & f_{2} & f_{3} & f_{4} & 0 & f_{6} & f_{7} & f_{8}
\end{array}\right]
$$


where

$$
\begin{aligned}
& f_{1}=1-\xi, \\
& f_{2}=1-10 \xi^{3}+15 \xi^{4}-6 \xi^{5}, \\
& f_{3}=l\left(\xi-6 \xi^{3}+8 \xi^{4}-3 \xi^{5}\right), \\
& f_{5}=\xi \\
& f_{4}=\frac{l^{2}\left(\xi^{2}-3 \xi^{3}+3 \xi^{4}-\xi^{5}\right)}{2}, \\
& f_{6}=10 \xi^{3}-15 \xi^{4}+6 \xi^{5}, \\
& f_{7}=l\left(-4 \xi^{3}+7 \xi^{4}-3 \xi^{5}\right), \\
& f_{8}=\frac{l^{2}\left(\xi^{3}-2 \xi^{4}+\xi^{5}\right)}{2} .
\end{aligned}
$$

$l$ is length of the beam element.

The deformation displacements of $C^{\prime}$ in the $A-x y$ can be written as [14]

$$
\mathbf{v}=\left[\begin{array}{l}
v_{1}(x, t) \\
v_{2}(x, t)
\end{array}\right]=\left[v_{10}-y \frac{\partial v_{20}}{\partial x}-\int_{v_{20}}^{x} \frac{1}{2}\left(\frac{\partial v_{20}}{\partial \xi}\right)^{2} d \xi\right] ;
$$

then, the displacement of $C^{\prime}$ can be expressed in the global fixed frame by

$$
\mathbf{r}_{e, C^{\prime}}=\mathbf{r}_{e, A}+\mathbf{R}\left(\mathbf{e}_{0}+\mathbf{v}\right),
$$

where $\mathbf{R}$ is the planar transformation matrix defined as

$$
\mathbf{R}=\left[\begin{array}{cc}
\cos \varphi & -\sin \varphi \\
\sin \varphi & \cos \varphi
\end{array}\right]
$$

$\mathbf{e}_{0}$ is the location coordinates vector of the point $C$ in the $A-x y$ system and is given by $\left[\begin{array}{ll}x & y\end{array}\right]^{T}$.

Taking the first derivative on (5) and considering (1), the velocity vector of the point $C$ in the $O-X Y$ system can be written as

$$
\begin{aligned}
& \dot{\mathbf{r}}_{e, C^{\prime}}=\dot{\mathbf{r}}_{e, A}+\mathbf{R}_{\varphi}\left(\mathbf{e}_{0}+\mathbf{N e}_{f}+\left[\begin{array}{c}
w_{c} \\
0
\end{array}\right]\right) \dot{\varphi} \\
& +\mathbf{R}\left(\mathbf{N}-\left[\begin{array}{c}
\mathbf{e}_{f}^{T} \mathbf{H}_{e} \\
\mathbf{0}
\end{array}\right]\right) \dot{\mathbf{e}}_{f}
\end{aligned}
$$



$$
\begin{aligned}
& \times\left[\begin{array}{c}
\dot{\mathbf{r}}_{e, A} \\
\dot{\varphi} \\
\dot{\mathbf{e}}_{f}
\end{array}\right] \\
& =\mathbf{S u} \text {, }
\end{aligned}
$$

where

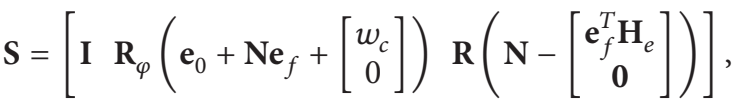

$$
\begin{aligned}
& \mathbf{u}=\left[\begin{array}{lll}
\mathbf{r}_{e, A}^{T} & \varphi & \mathbf{e}_{f}^{T}
\end{array}\right]^{T},
\end{aligned}
$$

$\dot{\mathbf{r}}_{e, A}$ is the velocity vector of the original point $A$ in the $O-X Y$ system,

$$
\begin{aligned}
& \mathbf{H}_{e}=\int_{0}^{x} \frac{\partial \overline{\mathbf{N}}_{2}}{\partial \xi} \frac{\partial \overline{\mathbf{N}}_{2}^{T}}{\partial \xi} d \xi, \\
& \mathbf{R}_{\varphi}=\frac{\partial \mathbf{R}}{\partial \varphi}, \quad \mathbf{N}=\left[\begin{array}{c}
\overline{\mathbf{N}}_{1}^{T}-y \frac{\partial \overline{\mathbf{N}}_{2}^{T}}{\partial x} \\
\overline{\mathbf{N}}_{2}^{T}
\end{array}\right],
\end{aligned}
$$

I is $2 \times 2$ unit matrix, and parameter $w_{c}=-(1 / 2) \mathbf{e}_{f}^{T} \mathbf{H}_{e} \mathbf{e}_{f}$ is the second-order coupling term that is the axial shrinking quantity caused by the transverse displacement $v_{2}$. In the ZOAC model, the small deformation assumption is adopted, so $v_{1}(x, t)=v_{10}-y\left(\partial v_{20} / \partial x\right)$ is taken, and $w_{c}$ is neglected.

2.1. Kinetic Energy of the Beam Element. Using (7), kinetic energy of the beam element which includes translational kinetic energy of the beam element, rotational kinetic energy of the cross-section, and kinetic energy of lumped mass at the end points of beam can be written as

$$
\begin{aligned}
T_{e}= & \frac{1}{2} \int_{V} \rho \dot{\mathbf{r}}_{e, C^{\prime}}^{T} \dot{\mathbf{r}}_{e, C^{\prime}} d V \\
& +\frac{1}{2} \int_{0}^{l} \dot{\theta}^{2}(x, t) d J_{c}+\frac{1}{2}\left(m_{L} \dot{\mathbf{r}}_{A^{\prime}}^{T} \dot{\mathbf{r}}_{A^{\prime}}+m_{R} \dot{\mathbf{r}}_{B^{\prime}}^{T} \dot{\mathbf{r}}_{B^{\prime}}\right) \\
& +\frac{1}{2}\left(J_{L} \dot{\theta}^{2}(0, t)+J_{R} \dot{\theta}^{2}(l, t)\right) \\
= & \frac{1}{2} \dot{\mathbf{u}}^{T} \mathbf{m}_{e} \dot{\mathbf{u}},
\end{aligned}
$$

where

$$
\begin{gathered}
\mathbf{m}_{e}=\left[\begin{array}{ccc}
\mathbf{m}_{e r r} & \mathbf{m}_{e r \varphi} & \mathbf{m}_{\text {erf }} \\
& m_{e \varphi \varphi} & \mathbf{m}_{e \varphi f} \\
\text { Symmetry } & & \mathbf{m}_{e f f}
\end{array}\right], \\
\mathbf{m}_{\text {err }}=\left(\int_{V} \rho d V+m_{L}+m_{R}\right) \mathbf{I},
\end{gathered}
$$




$$
\begin{aligned}
& \mathbf{m}_{e r \varphi}=\mathbf{R}_{\varphi} \mathbf{N}_{1}+\mathbf{R}_{\varphi} \mathbf{N}_{2} \mathbf{e}_{f}+\mathbf{R}_{\varphi} \int_{V} \rho\left[\begin{array}{c}
w_{c} \\
0
\end{array}\right] d V+\mathbf{R}_{\varphi} \overline{\mathbf{N}}_{c} \mathbf{e}, \\
& \mathbf{m}_{e r f}=\mathbf{R N}_{2}-\mathbf{R} \int_{V} \rho\left[\begin{array}{c}
\mathbf{e}_{f}^{T} \mathbf{H}_{e} \\
\mathbf{0}
\end{array}\right] d V+\mathbf{R} \overline{\mathbf{N}}_{c}, \\
& m_{e \varphi \varphi}=\mathbf{N}_{3}+2 \mathbf{N}_{4} \mathbf{e}_{f}+\mathbf{e}_{f}^{T} \mathbf{N}_{8} \mathbf{e}_{f} \\
& +\mathbf{e}_{f}^{T} \mathbf{N}_{5} \mathbf{e}_{f}+\rho I l+\mathbf{e}^{T} \widetilde{\mathbf{N}}_{c} \mathbf{e}+J_{L}+J_{R}, \\
& \mathbf{m}_{e \varphi f}=\mathbf{N}_{6}+\mathbf{e}_{f}^{T} \mathbf{N}_{7}+\mathbf{e}_{f}^{T} \mathbf{N}_{9}+\mathbf{e}^{T} \widetilde{\mathbf{N}}_{c}+\mathbf{C}_{1} \text {, } \\
& \mathbf{m}_{e f f}=\mathbf{N}_{5}+\mathbf{C}_{2} \text {, } \\
& \mathbf{N}_{1}=\int_{V} \rho \mathbf{e}_{0} d V \\
& \mathbf{N}_{2}=\int_{V} \rho \mathbf{N} d V, \quad \mathbf{N}_{3}=\int_{V} \rho \mathbf{e}_{0}^{T} \mathbf{e}_{0} d V, \\
& \mathbf{N}_{4}=\int_{V} \rho \mathbf{e}_{0}^{T} \mathbf{N} d V, \\
& \mathbf{N}_{5}=\int_{V} \rho \mathbf{N}^{T} \mathbf{N} d V, \quad \mathbf{N}_{6}=\int_{V} \rho \mathbf{e}_{0}^{T} \widetilde{\mathbf{I}} \mathbf{N} d V, \\
& \mathbf{N}_{7}=\int_{V} \rho \mathbf{N}^{T} \widetilde{\mathbf{I}} \mathbf{N} d V, \\
& \mathbf{N}_{8}=-\int_{V} \rho x \mathbf{H}_{e} d V, \quad \mathbf{N}_{9}=\int_{V} \rho y \mathbf{H}_{e} d V, \\
& \overline{\mathbf{N}}_{c}=m_{L} \mathbf{N}(0)+m_{R} \mathbf{N}(l), \\
& \widetilde{\mathbf{N}}_{c}=m_{L} \mathbf{N}(0)^{T} \mathbf{N}(0)+m_{R} \mathbf{N}(l)^{T} \mathbf{N}(l), \\
& \widetilde{\overline{\mathbf{N}}}_{c}=m_{L} \mathbf{N}(0)^{T} \widetilde{\mathbf{I}} \mathbf{N}(0)+m_{R} \mathbf{N}(l)^{T} \widetilde{\mathbf{I}} \mathbf{N}(l), \\
& \mathbf{C}_{1}=\int_{0}^{l} \rho I \mathbf{N}_{2}^{\prime T} d x+J_{L} \mathbf{N}_{2}^{\prime T}(0)+J_{R} \mathbf{N}_{2}^{\prime T}(l), \\
& \mathbf{C}_{2}=\int_{0}^{l} \rho I \mathbf{N}_{2}^{\prime} \mathbf{N}_{2}^{\prime T} d x+\widetilde{\mathbf{N}}_{c} \\
& +J_{L} \mathbf{N}_{2}^{\prime}(0) \mathbf{N}_{2}^{\prime T}(0)+J_{R} \mathbf{N}_{2}^{\prime}(l) \mathbf{N}_{2}^{\prime T}(l) .
\end{aligned}
$$

$\widetilde{\mathbf{I}}$ is skew symmetric matrix, $\theta(x, t)=\varphi+\partial v_{2}(x, t) / \partial x$ is absolute rotation angle of microsegment $d x$ which distances $x$ from the original point $A . m_{L}, J_{L}, m_{R}$, and $J_{R}$ are lumped mass and lumped moment of inertia at left and right end points of the beam element, respectively. $\mathbf{N}_{2}^{\prime}=\partial \mathbf{N}_{2} / \partial x$. It should be mentioned that the high-order terms related with $w_{c}$, such as $v_{10} w_{c}, \dot{w}_{c}^{2}, v_{2} \dot{w}_{c}$, and $\dot{v}_{2} w_{c}$, are omitted since $w_{c}$, $\dot{w}_{c}, v_{10}, v_{2}$, and $\dot{v}_{2}$ are very small.

2.2. Strain Energy of the Beam Element. Nonlinear terms in the strain-displacement relationship are neglected [16]. Then, strain energy of the beam element can be written as

$$
\begin{aligned}
U_{e}= & \frac{1}{2} \int_{0}^{l} E I(x)\left(v_{20}^{\prime \prime}(x, t)\right)^{2} d x \\
& +\frac{1}{2} \int_{0}^{l} E S(x)\left(v_{10}^{\prime}(x, t)\right)^{2} d x \\
= & \frac{1}{2} \mathbf{u}^{T} \mathbf{k}_{e} \mathbf{u},
\end{aligned}
$$

where $v_{10}^{\prime}(x, t)=\partial v_{10}(x, t) / \partial x, v_{20}^{\prime \prime}(x, t)=\partial^{2} v_{20}(x, t) / \partial x^{2}$, and $E$ is elastic modulus of materials. $I(x), S(x)$ are crosssectional moment of inertia and cross-sectional area of the beam element, respectively. Consider

$$
\begin{aligned}
\mathbf{k}_{\mathbf{e}}= & {\left[\begin{array}{lll}
\mathbf{0} & \mathbf{0} & \mathbf{0} \\
\mathbf{0} & \mathbf{0} & \mathbf{0} \\
\mathbf{0} & \mathbf{0} & \mathbf{k}_{\text {eff }}
\end{array}\right], } \\
\mathbf{k}_{e f f}= & \int_{0}^{l} E I(x) \mathbf{N}_{2}^{\prime{ }^{T}}(x) \mathbf{N}_{2}^{\prime \prime}(x) d x \\
& +\int_{0}^{l} E S(x) \mathbf{N}_{1}^{\prime T}(x) \mathbf{N}_{1}^{\prime}(x) d x .
\end{aligned}
$$

2.3. Dynamic Equation of the Beam Element. According to Lagrange's equation, dynamic equation of the beam element can be written as

$$
\mathbf{m}_{e} \ddot{\mathbf{u}}+\mathbf{k}_{e} \mathbf{u}=\mathbf{p}_{e, e}+\mathbf{p}_{e, v},
$$

where $\mathbf{p}_{e, e}$ and $\mathbf{p}_{e, v}$ are the generalized external forces and the quadratic velocity vector that contains the gyroscopic and the Coriolis force components, respectively.

The elemental dynamic equation has been established in the A-xy system. Before forming the dynamic equation of the system, (14) must be expressed in the $O-X Y$ system. Define the coordinate transformation matrix $\mathbf{B}$ as follows:

$$
\mathbf{B}=\left[\begin{array}{cccccccc}
\cos \varphi & \sin \varphi & 0 & 0 & 0 & 0 & 0 & 0 \\
-\sin \varphi & \cos \varphi & 0 & 0 & 0 & 0 & 0 & 0 \\
0 & 0 & 1 & 0 & 0 & 0 & 0 & 0 \\
0 & 0 & 0 & 1 & 0 & 0 & 0 & 0 \\
0 & 0 & 0 & 0 & \cos \varphi & \sin \varphi & 0 & 0 \\
0 & 0 & 0 & 0 & -\sin \varphi & \cos \varphi & 0 & 0 \\
0 & 0 & 0 & 0 & 0 & 0 & 1 & 0 \\
0 & 0 & 0 & 0 & 0 & 0 & 0 & 1
\end{array}\right] .
$$

Let

$$
\mathbf{e}_{f}=\mathbf{B E}_{e},
$$

where $\mathbf{E}_{e}$ is the elemental nodal coordinate vector in the $O-X Y$ system. Taking the first and the second derivative on (16) with respect to time yields

$$
\begin{gathered}
\dot{\mathbf{e}}_{f}=\mathbf{B}_{\varphi} \mathbf{E}_{e} \dot{\varphi}+B \dot{\mathbf{E}}_{e}, \\
\ddot{\mathbf{e}}_{f}=\mathbf{B}_{\varphi \varphi} \mathbf{E}_{e} \dot{\varphi}^{2}+2 \mathbf{B}_{\varphi} \dot{\mathrm{E}}_{e} \dot{\varphi}+\mathbf{B}_{\varphi} \mathbf{E}_{e} \ddot{\varphi}+\mathbf{B} \ddot{\mathbf{E}}_{e},
\end{gathered}
$$

where $\mathbf{B}_{\varphi}=\partial \mathbf{B} / \partial \varphi, \mathbf{B}_{\varphi \varphi}=\partial^{2} \mathbf{B} / \partial \varphi^{2}$. Substitute(16) and (17) into (14) and premultiply by matrix

$$
\left[\begin{array}{ccc}
\mathbf{I} & \mathbf{0} & \mathbf{0} \\
\mathbf{0} & 1 & \mathbf{E}_{e}^{T} \mathbf{B}_{\varphi} \\
\mathbf{0} & \mathbf{0} & \mathbf{B}^{T}
\end{array}\right] .
$$

Then, dynamic equation of the beam element can be expressed in the $O-X Y$ system by

$$
\mathbf{M}_{e} \ddot{\mathbf{U}}_{e}+\mathbf{K}_{e} \mathbf{U}_{e}=\mathbf{Q}_{e, e}+\mathbf{Q}_{e, v},
$$




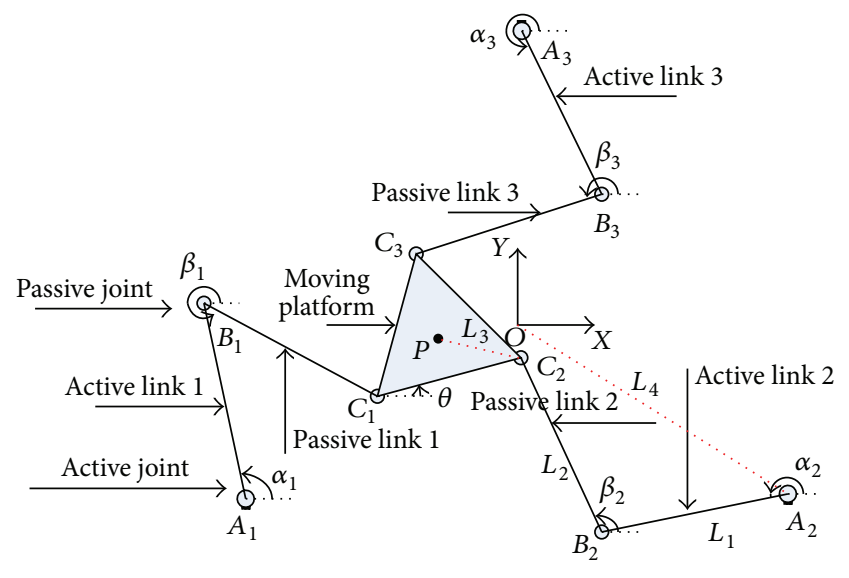

FIGURE 2: The sketch of planar 3-RRR parallel robot.

where

$$
\mathbf{U}_{e}=\left[\begin{array}{lll}
\mathbf{r}_{e, A}^{T} & \varphi & \mathbf{E}_{e}^{T}
\end{array}\right]^{T}
$$

where $\mathbf{M}_{e}$ and $\mathbf{K}_{e}$ are $8 \times 8$ the element generalized mass and stiffness matrixes in the $O-X Y$ system, respectively. $\mathbf{Q}_{e, v}$ is $8 \times 1$ the quadratic velocity vector in the $O-X Y$ system. $\mathbf{Q}_{e, e}$ is $8 \times 1$ the generalized external forces vector in the $O-X Y$ system.

\section{Governing Dynamic Equation of Planar 3-RRR Flexible Parallel Robots}

In this section, according to the above dynamic equation of the beam element, governing dynamic equation of the planar 3-RRR parallel robots can be established, in which constraint equations are considered.

3.1. The Sketch of Planar 3-RRR Parallel Robots. The sketch of planar flexible 3-RRR parallel robots is constructed by the regular triangle moving platform $C_{1} C_{2} C_{3}$, the static platform, and three symmetrical kinematic chains $A_{1} B_{1} C_{1}, A_{2} B_{2} C_{2}$, and $A_{3} B_{3} C_{3}$, as shown in Figure 2. Each kinematic chain has one active revolute $(\underline{R})$ joint followed by two consecutive passive revolute $(\mathrm{R})$ joints. The active revolute joints are installed at $A_{i}, i=1,2,3 . A_{1}, A_{2}$ and $A_{3}$ are the regular triangle's three vertices. $A_{1} B_{1}=A_{2} B_{2}=A_{3} B_{3}$, and $B_{1} C_{1}=$ $B_{2} C_{2}=B_{3} C_{3}$. The vertexes $O$ and $P$ are centers of the regular triangles $A_{1} A_{2} A_{3}$ and $C_{1} C_{2} C_{3}$, respectively. The $O-X Y$ is the global fixed frame. Parameters $\alpha_{i}, \beta_{i}(i=1,2,3)$ are the angles between the $X$-axis of the fixed frame and linkages $A_{i} B_{i}, B_{i} C_{i}(i=1,2,3)$, respectively. $\theta$ is the angle between the $X$-axis of the fixed frame and side $C_{1} C_{2}$ of the regular triangle $C_{1} C_{2} C_{3} . L_{1}, L_{2}, L_{3}$, and $L_{4}$ are the length of the segments $A_{2} B_{2}, B_{2} C_{2}, C_{2} P$, and $O A_{2}$.

3.2. Constraint Equations. Different from the simple structure, constraint relationships of planar $3-\mathrm{RRR}$ of flexible parallel robots which include the rigid body motion constraints, the elastic deformation motion constraints, and dynamic constraints of the moving platform are very complex and can be used to eliminate the correlation of generalized coordinates.

3.2.1. Constraint Equations of the Rigid-Body Motion. Generalized coordinates are formed by rigid-body motion coordinates and elastic coordinates. As shown in Figure 2, rigidbody motion coordinates that include drive joint rotation angles $\boldsymbol{\alpha}=\left[\begin{array}{lll}\alpha_{1} & \alpha_{2} & \alpha_{3}\end{array}\right]^{T}$, passive joint rotation angles $\boldsymbol{\beta}=$ $\left[\begin{array}{lll}\beta_{1} & \beta_{2} & \beta_{3}\end{array}\right]^{T}$, translation displacement and rotation angle of the moving platform $\mathbf{X}_{P}=\left[\begin{array}{lll}X_{P} & Y_{P} & \theta\end{array}\right]^{T}$, and rigid body motion coordinate vector $\boldsymbol{\alpha}, \boldsymbol{\beta}, \mathbf{X}_{P}$ are not independent. Because three kinematic chains of 3 -RRR parallel robots are full symmetrical, one chain is studied. As shown in Figure 3, a closed-loop vector equation can be established

$$
\overrightarrow{\mathrm{OA}_{i}}+\overrightarrow{A_{i} B_{i}}+\overrightarrow{B_{i} C_{i}}+\overrightarrow{C_{i} P}-\overrightarrow{O P}=\overrightarrow{0}
$$

Projecting (21) into $X$ and $Y$ component yields

$$
\begin{array}{r}
X_{A_{i}}+L_{1} \cos \alpha_{i}+L_{2} \cos \beta_{i} \\
+L_{3} \cos \left(\frac{\pi}{6}+\frac{2(i-1) \pi}{3}+\theta\right)-X_{P}=0, \\
Y_{A_{i}}+L_{1} \sin \alpha_{i}+L_{2} \sin \beta_{i} \\
+L_{3} \sin \left(\frac{\pi}{6}+\frac{2(i-1) \pi}{3}+\theta\right)-Y_{P}=0, \\
i=1,2,3,
\end{array}
$$

where $\left(X_{A_{i}}, Y_{A_{i}}\right)$ and $\left(X_{P}, Y_{P}\right)$ are the coordinates of the points $A_{i}$ and $P$ in the $O-X Y$ system, respectively.

3.2.2. Constraint Equations of the Elastic Deformation Motion. As shown in Figure 3, center point $P$ and vertex $C_{i}$ of the moving platform are moved to the points $P^{\prime}, C_{i}^{\prime}$ because of elastic deformation motion of the flexible links $A_{i} B_{i}$, $B_{i} C_{i}(i=1,2,3)$. Setting $P-x y$ is the local coordinate system with original point $P$, and $P^{\prime}-x^{\prime} y^{\prime}$ is the elastic coordinate system with original point $P^{\prime}$. T, $\widetilde{\mathbf{T}}$ and $\overline{\mathbf{T}}$ are the coordinate transformation matrixes for $p$ - $x y \rightarrow O-X Y, p^{\prime}-x^{\prime} y^{\prime} \rightarrow$ $p-x y$ and $p^{\prime}-x^{\prime} y^{\prime} \rightarrow O-X Y$, respectively. Then, $\overline{\mathbf{T}}=\mathbf{T} \widetilde{\mathbf{T}}$. $U_{i_{22}}, U_{i_{23}}, U_{i_{24}}$ are elastic displacements and elastic rotational angle at the end point $C_{i}$ of the flexile link $B_{i} C_{i}$, and the section curvature is equal to zero at the point $C_{i} . \Delta X_{P}$, and $\Delta Y_{P}, \mathcal{E}$ are translational and rotational declinations of the moving platform because of elastic vibration of the flexible links. Assuming that the moving platform is rigid, so $P C_{i}=$ $P^{\prime} C_{i}^{\prime}$. Noting that

$$
\mathbf{T}=\left[\begin{array}{ccc}
\cos \theta & -\sin \theta & X_{P} \\
\sin \theta & \cos \theta & Y_{P} \\
0 & 0 & 1
\end{array}\right], \quad \widetilde{\mathbf{T}}=\left[\begin{array}{ccc}
1 & -\varepsilon & \Delta X_{P} \\
\varepsilon & 1 & \Delta Y_{P} \\
0 & 0 & 1
\end{array}\right]
$$




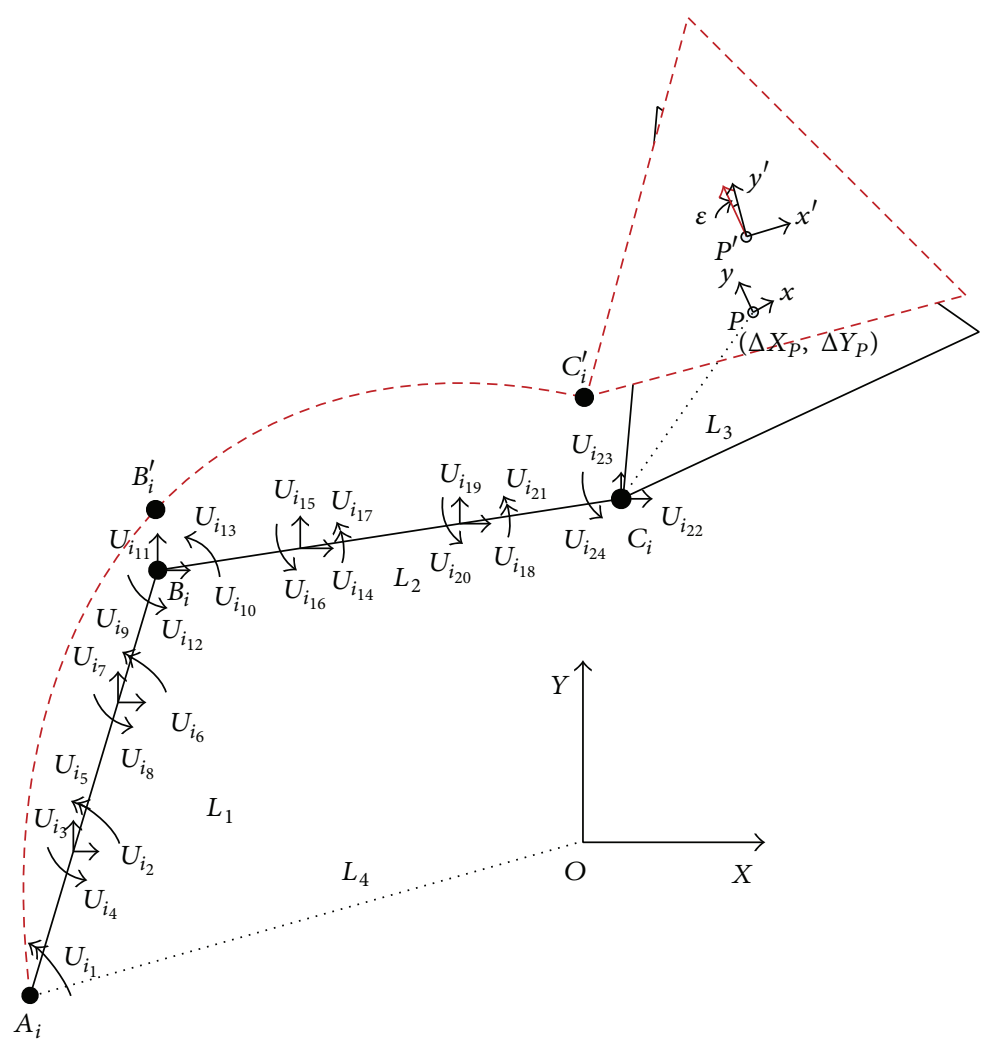

Figure 3: The flexible kinematic chain.

Assuming that $\left(\begin{array}{lll}X_{C_{i}} & Y_{C_{i}}\end{array}\right)$ and $\left(\begin{array}{lll}X_{C_{i}^{\prime}} & Y_{C_{i}^{\prime}}\end{array}\right)$ are the coordinates of the points $C_{i}$ and $C_{i}^{\prime}$ in the $O-X Y$ system yields

$$
\begin{aligned}
& {\left[\begin{array}{c}
X_{C_{i}^{\prime}} \\
Y_{C_{i}^{\prime}} \\
1
\end{array}\right]=\overline{\mathbf{T}}\left[\begin{array}{c}
x_{C_{i}^{\prime}}^{\prime} \\
y_{C_{i}^{\prime}}^{\prime} \\
1
\end{array}\right]_{P^{\prime}},} \\
& {\left[\begin{array}{c}
X_{C_{i}} \\
Y_{C_{i}} \\
1
\end{array}\right]=\mathbf{T}\left[\begin{array}{c}
x_{C_{i}} \\
y_{C_{i}} \\
1
\end{array}\right]_{P},}
\end{aligned}
$$

where $\left(x_{C_{i}}, y_{C_{i}}\right)_{p}=\left(x_{C_{i}^{\prime}}^{\prime}, y_{C_{i}^{\prime}}^{\prime}\right)_{p^{\prime}}=\left(L_{3} \cos (\pi / 2+\right.$ $\left.2 i \pi / 3), L_{3} \sin (\pi / 2+2 i \pi / 3)\right)$. ( ) $p$ means the description in the $p-x y$.

Setting

$$
\mathbf{T}_{1}=\left[\begin{array}{cc}
\cos \theta & -\sin \theta \\
\sin \theta & \cos \theta
\end{array}\right]
$$

yields

$$
\begin{aligned}
& {\left[\begin{array}{c}
U_{i_{22}} \\
U_{i_{23}}
\end{array}\right]=\left[\begin{array}{c}
X_{C_{i}^{\prime}} \\
Y_{C_{i}^{\prime}}^{\prime}
\end{array}\right]-\left[\begin{array}{c}
X_{C_{i}} \\
Y_{C_{i}}
\end{array}\right]=\left[\begin{array}{ll}
\mathbf{I} & \mathbf{T}_{1}\left(\begin{array}{c}
-y_{C_{i}} \\
x_{C_{i}}
\end{array}\right)_{P}
\end{array}\right]\left[\begin{array}{c}
\Delta X_{P} \\
\Delta Y_{P} \\
\varepsilon
\end{array}\right]} \\
& \varepsilon=\sum_{i=1}^{3} U_{i_{24}}
\end{aligned}
$$

where $\mathbf{I}$ is $2 \times 2$ unit matrix.
3.2.3. Dynamic Constraints of the Moving Platform. Assuming that $\mathbf{F}_{i}$ is the generalized joint constraint antiforce that the passive joint $C_{i}$ are applied to the moving platform. $M_{v}, J_{v}$ are the mass and moment of inertia of the moving platform. Then, dynamic constraint of the moving platform can be expressed by

$$
\left[\begin{array}{ccc}
M_{v} & & \\
& M_{v} & \\
& & J_{v}
\end{array}\right]\left[\begin{array}{c}
\Delta \ddot{X}_{P} \\
\Delta \ddot{Y}_{P} \\
\ddot{\varepsilon}
\end{array}\right]=\sum_{i=1}^{3} \mathbf{F}_{i}-\left[\begin{array}{c}
M_{v} \ddot{X}_{P} \\
M_{v} \ddot{Y}_{P} \\
J_{v} \ddot{\varepsilon}
\end{array}\right] .
$$

\section{Governing Dynamic Equation of the Flexible Robots System}

Considering constraint equations ((22)-(27)) and assembling all the element dynamic equations with respect to the compatibility at the nodes, then the governing dynamic equation that describes the dynamic characteristic of system can be formed as

$$
\mathbf{M U}+\mathbf{K U}=\mathbf{Q}_{e}+\mathbf{Q}_{v}
$$

where $\mathbf{U}$ is the generalized coordinate vector of planer 3-RRR flexible parallel robots in the $O-X Y$ system which includes rigid-body motion coordinates, node elastic deformation coordinates, and the declinations of the moving platform. Equation (28) is the nonlinear and rigid-flexible coupling 


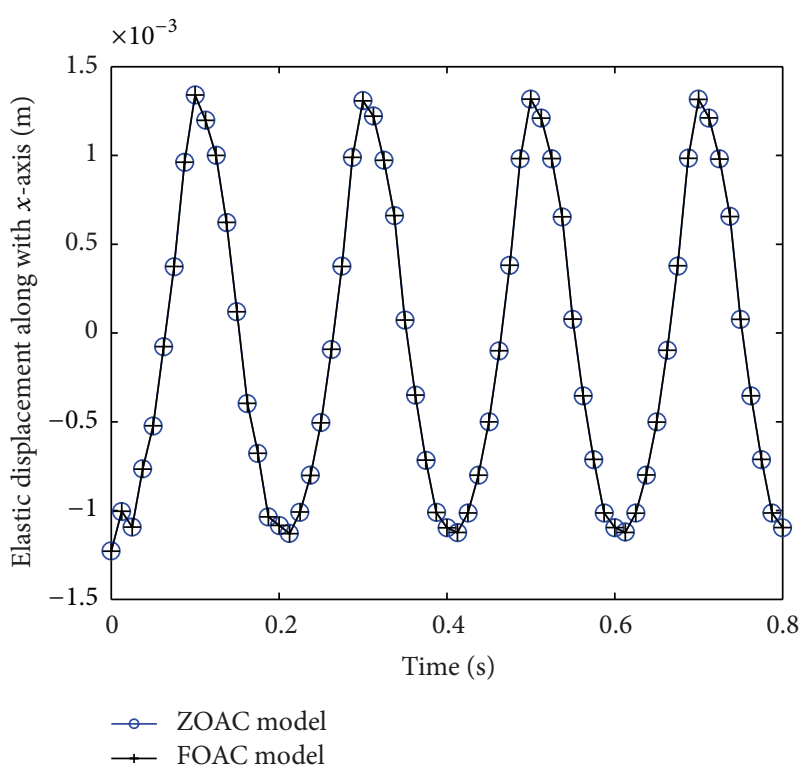

(a)

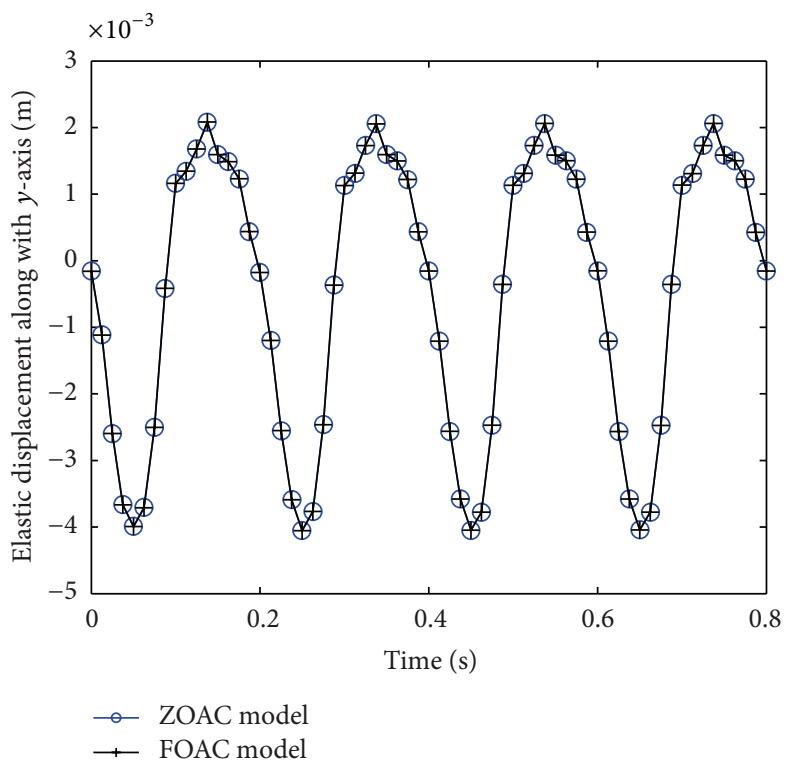

(b)

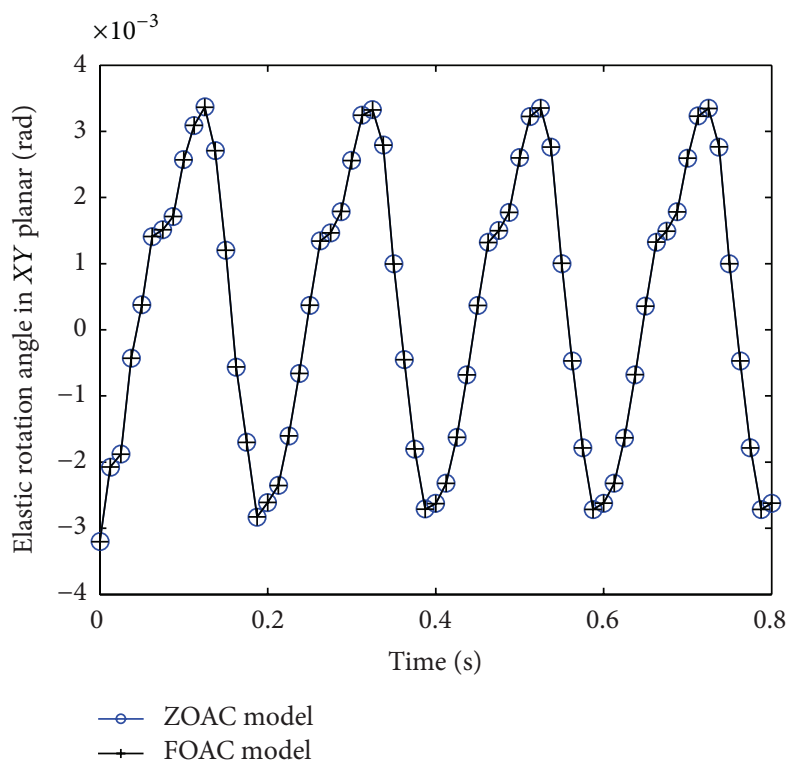

(c)

FIGURE 4: (a) and (b) are elastic displacements of the moving platform along with $X, Y$ direction, respectively; (c) is elastic rotational angle of the moving platform in $X Y$ planar, when $w=10 \pi \mathrm{rad} / \mathrm{s}$.

differential equation and can be decomposed into the rigid subsection and the flexible subsection as

$$
\begin{aligned}
\mathbf{K}(\boldsymbol{\alpha}, \dot{\boldsymbol{\alpha}}, \ddot{\boldsymbol{\alpha}}) & \\
=\sum_{e} \mathbf{D}_{e}^{T} \mathbf{B}^{T}\{ & {\left[\left(\mathbf{N}_{5}+\mathbf{C}_{2}\right) \mathbf{B}_{\varphi \varphi}-2\left(\mathbf{N}_{7}+\widetilde{\mathbf{N}}_{c}\right) \mathbf{B}_{\varphi}\right.} \\
& \left.-\left(\mathbf{N}_{5}+\widetilde{\mathbf{N}}_{c}\right) \mathbf{B}\right] \dot{\varphi}^{2} \\
& +\left[\left(\mathbf{N}_{5}+\mathbf{C}_{2}\right) \mathbf{B}_{\varphi}+\left(\mathbf{N}_{7}^{T}+\widetilde{\mathbf{N}}_{c}^{T}\right) \mathbf{B}\right] \ddot{\varphi} \\
& \left.+\mathbf{k}_{f f} \mathbf{B}\right\} \mathbf{D}_{e}
\end{aligned}
$$$$
\mathbf{M}_{f}(\boldsymbol{\alpha}) \ddot{\mathbf{U}}+\mathbf{C}_{f}(\boldsymbol{\alpha}, \dot{\boldsymbol{\alpha}}) \dot{\mathbf{U}}+\mathbf{K}_{f}(\boldsymbol{\alpha}, \dot{\boldsymbol{\alpha}}, \ddot{\boldsymbol{\alpha}}) \mathbf{U}=\mathbf{Q}_{f}(\boldsymbol{\alpha}, \dot{\boldsymbol{\alpha}}, \ddot{\boldsymbol{\alpha}}) .
$$

Now, one will emphatically study flexible subsection (30), in which generalized stiffness matrix $\mathbf{K}_{f}(\boldsymbol{\alpha}, \dot{\boldsymbol{\alpha}}, \ddot{\boldsymbol{\alpha}})$ can be expressed by 


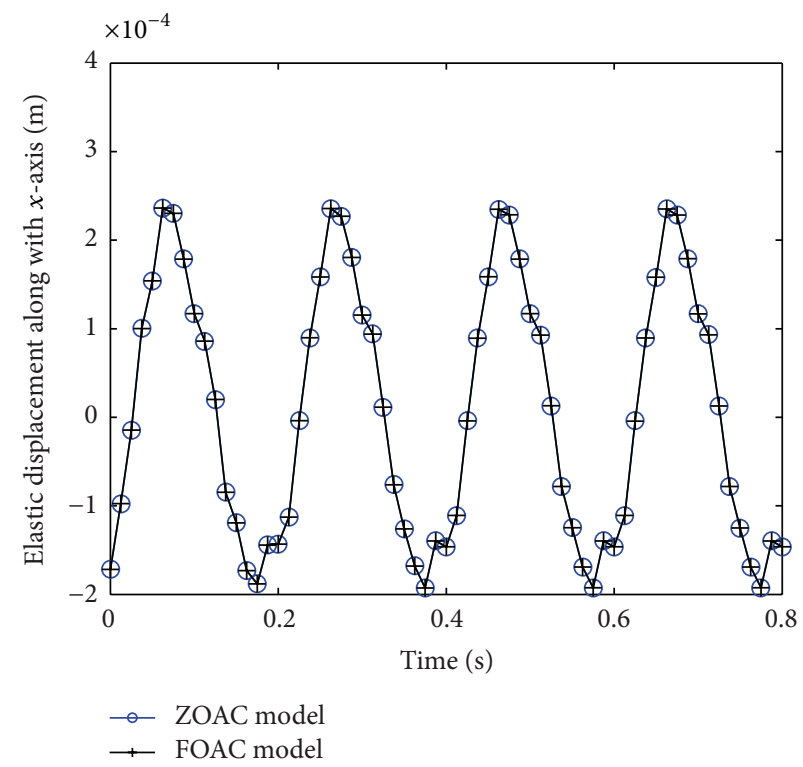

(a)

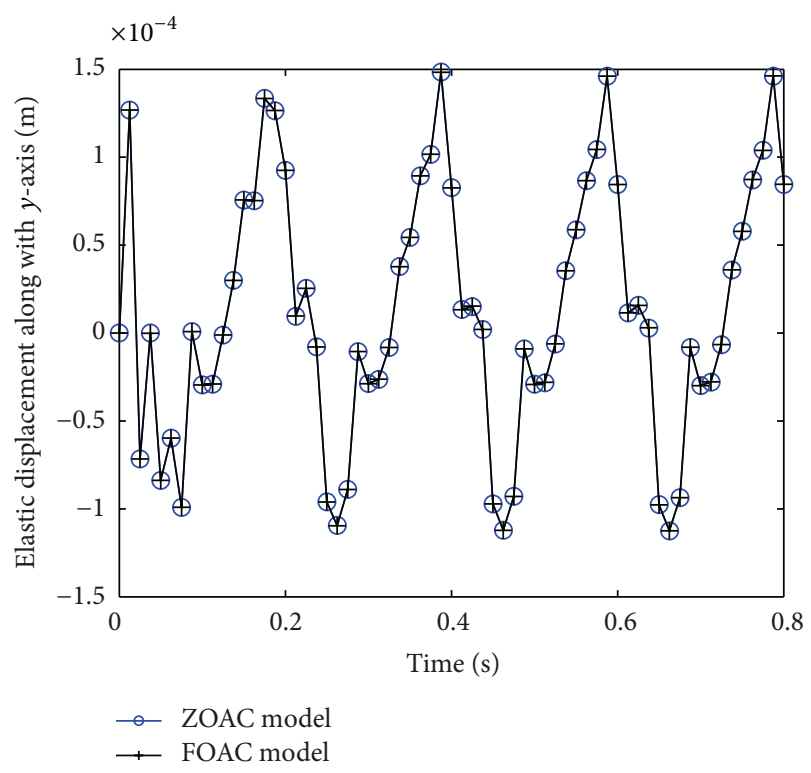

(b)

FIGURE 5: (a) and (b) are elastic displacements of along with $X, Y$ direction which are located at the 1/3 length of the active link 1 , respectively, when $w=10 \pi \mathrm{rad} / \mathrm{s}$.
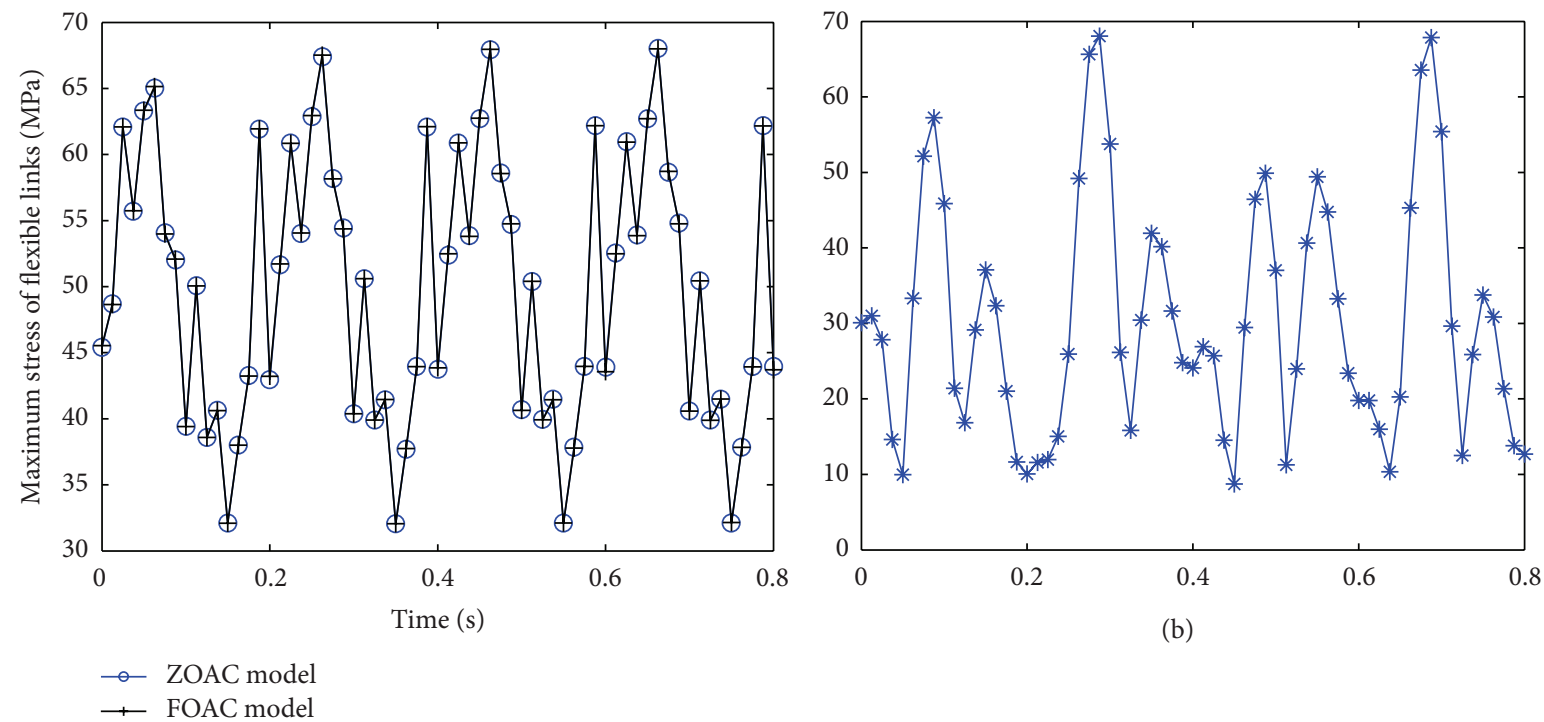

(a)

FIGURE 6: (a) is maximum stress response of all flexible links through numerical calculation and (b) is ANSYS results, when $w=10 \pi \mathrm{rad} / \mathrm{s}$.

$$
\begin{aligned}
& +\sum_{e} \mathbf{D}_{e}^{T} \mathbf{B}^{T}\left(g_{1 e} \widetilde{\mathbf{H}}_{e}+\mathbf{N}_{9}^{T} \ddot{\varphi}-\mathbf{N}_{8} \dot{\varphi}^{2}\right) \mathbf{B} \mathbf{D}_{e} \\
= & \mathbf{K}_{1}+\mathbf{K}_{d},
\end{aligned}
$$

where $\widetilde{\mathbf{H}}_{e}=\int_{V}-\rho \mathbf{H}_{e}^{T} d V, \mathbf{R}^{T} \ddot{\mathbf{r}}_{e, A}=\left[\begin{array}{l}g_{1 e} \\ g_{2 e}\end{array}\right], \mathbf{D}_{e}$ is the coordinate compatibility matrix, $\sum_{e}$ means summing to all elements, and $\varphi$ is a function of rigid body motion variable $\boldsymbol{\alpha}$. The underline term is the dynamic stiffening term [14]. In the conventional
ZOAC model, the term is equal to zero. In general, when the system operates at a high-speed and high-acceleration such as the flexible hub-beam [14-22], the term cannot be neglected.

\section{Numerical Simulation}

In this section, dynamic responses of planar 3-RRR flexible parallel robots are studied using the ZOAC model and FOAC model, comparing influences of the dynamic stiffening term 




(a)

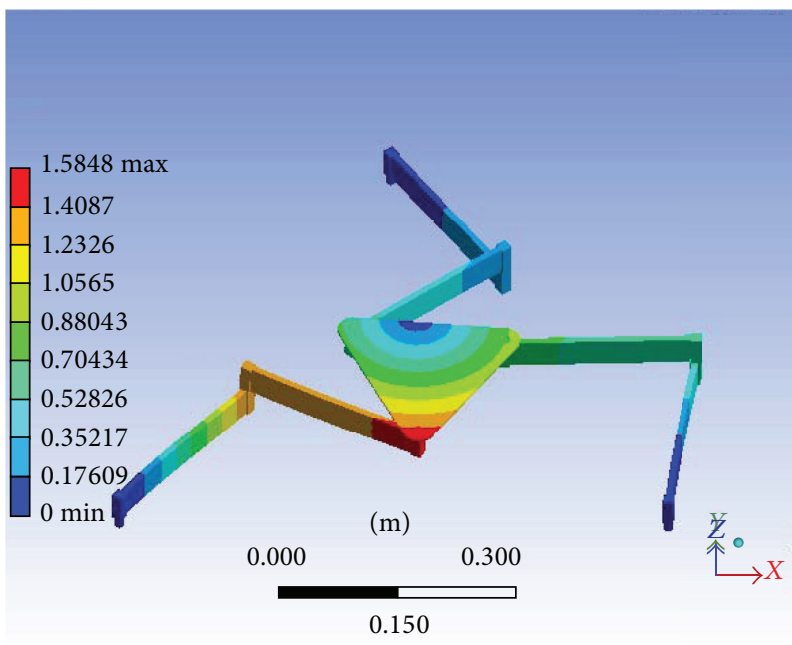

(c)

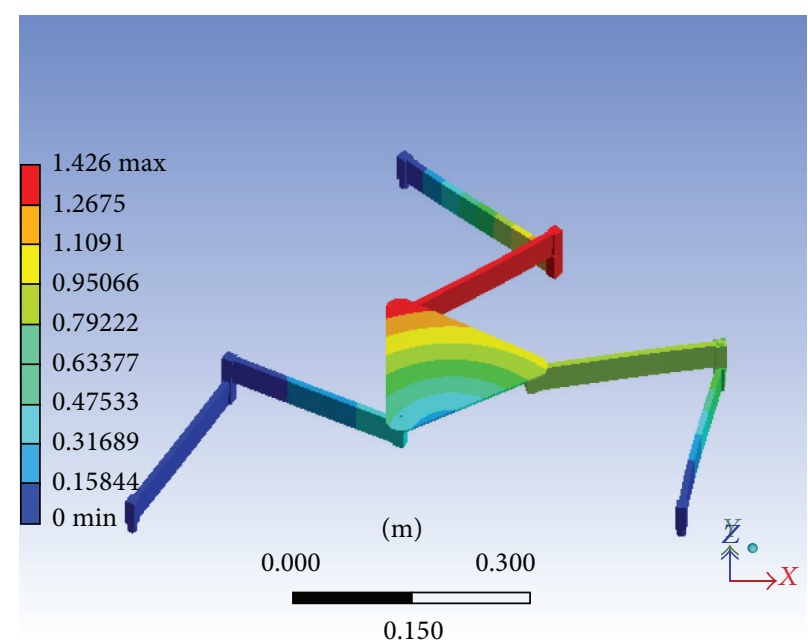

(b)

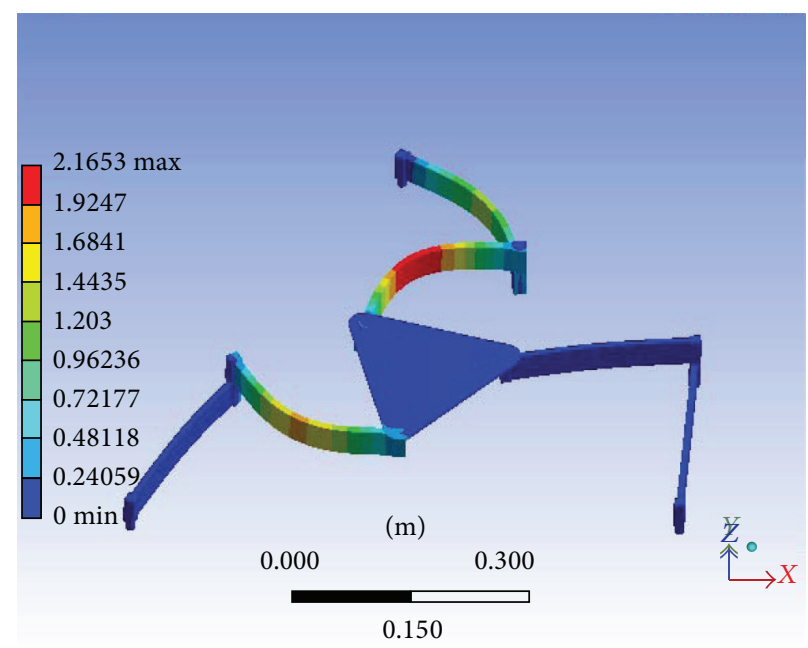

(d)

Figure 7: (a), (b), (c), and (d) are the first four-order modal shapes of the system, respectively.

TABLE 1: Parameters of the flexible links of 3-RRR.

\begin{tabular}{lcc}
\hline Parameter & Link $A_{i} B_{i}$ & $\operatorname{link} B_{i} C_{i}$ \\
\hline Length $(\mathrm{m})$ & 0.245 & 0.242 \\
Width $(\mathrm{m})$ & 0.025 & 0.025 \\
Thickness $(\mathrm{m})$ & 0.003 & 0.003 \\
Density $\rho\left(\mathrm{kg} / \mathrm{m}^{3}\right)$ & 2712 & \\
Young's Modulus $(\mathrm{Pa}) E$ & $7.102 \times 10^{10}$ & \\
Poisson's Ratio $\mu$ & 0.3 &
\end{tabular}

on dynamic response under the different speeds of the moving platform.

Assuming that all links that include three active links and three passive links are flexible and that other parts are rigid, every flexible link is divided into three equal length beam elements. Assume that the material of 3-RRR system is the aluminum alloy, the thickness of the moving platform $c=0.0034 \mathrm{~m}, L_{1}=0.245 \mathrm{~m}, L_{2}=0.242 \mathrm{~m}, L_{3}=0.108 \mathrm{~m}$, $L_{4}=0.4 \mathrm{~m}$, the lumped mass of the joint $M_{v}=0.02 \mathrm{~kg}$, and the lumped moment of inertia $J_{v}=0.00005 \mathrm{kgm}^{2}$. The other parameters of flexible links are defined as in Table 1.

It is assumed that the trajectory of the moving platform is described as

$$
\begin{gathered}
X_{P}=0.04 \cos (w \pi t)-0.02, \\
Y_{P}=0.04 \sin (w \pi t), \\
\theta=\frac{\pi}{4},
\end{gathered}
$$

where $w$ is angular frequency that can reflect moving speed of the motion platform. Due to the requirement of following numerical simulation, the parameter $w$ is chosen to be 


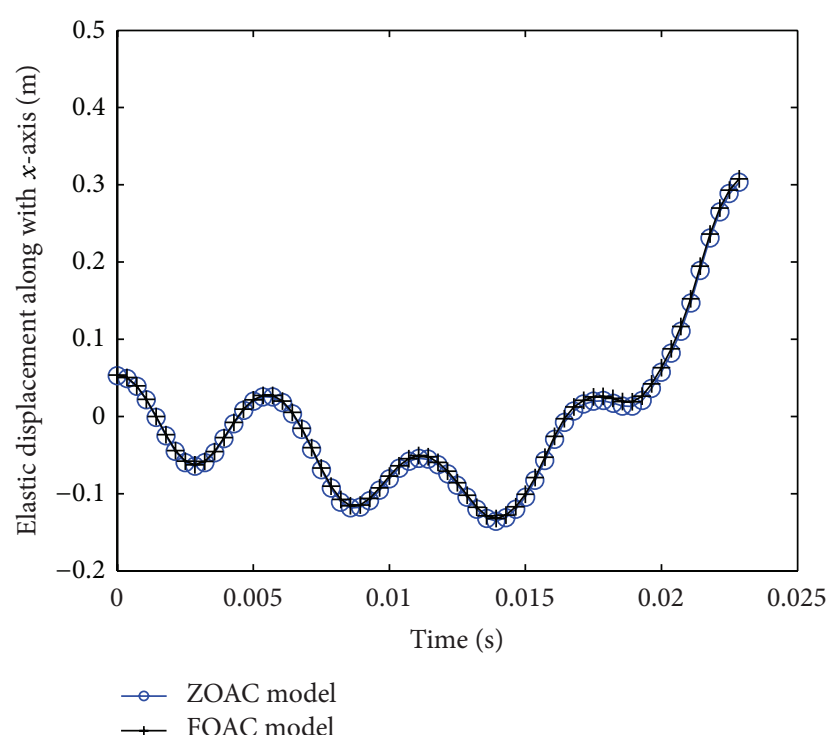

(a)

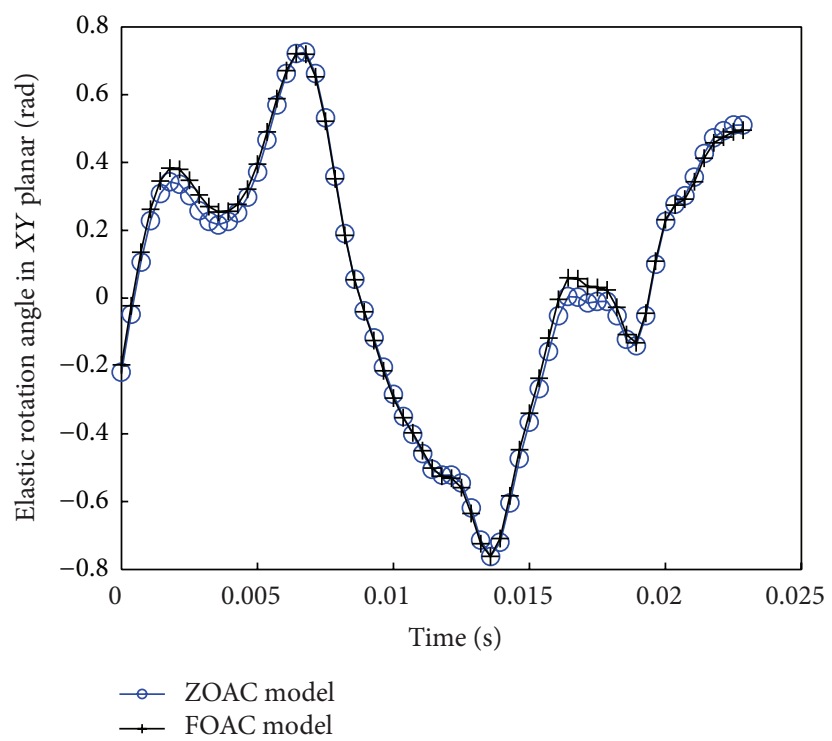

(c)

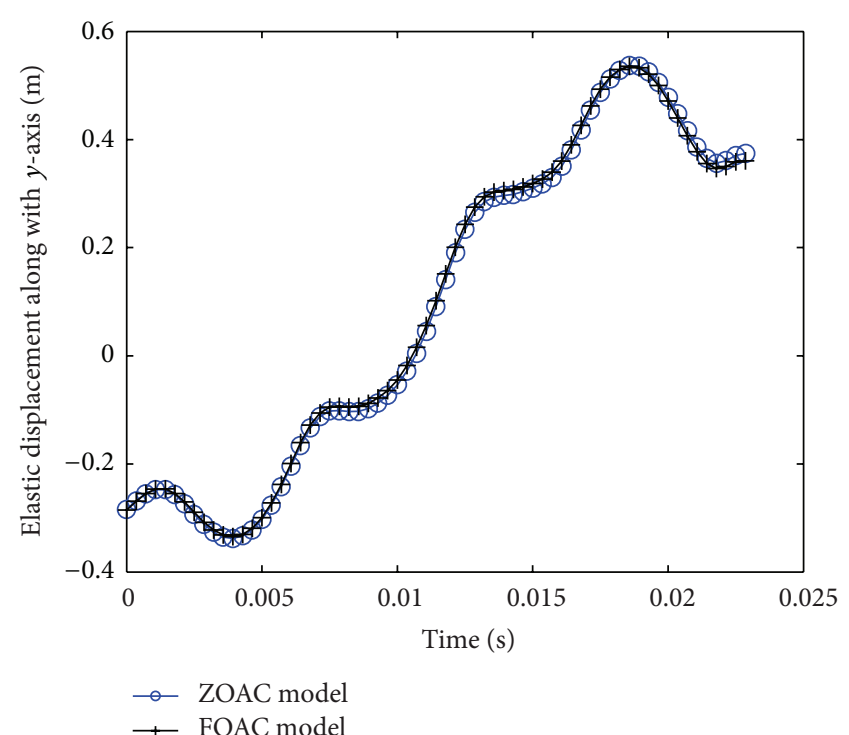

(b)

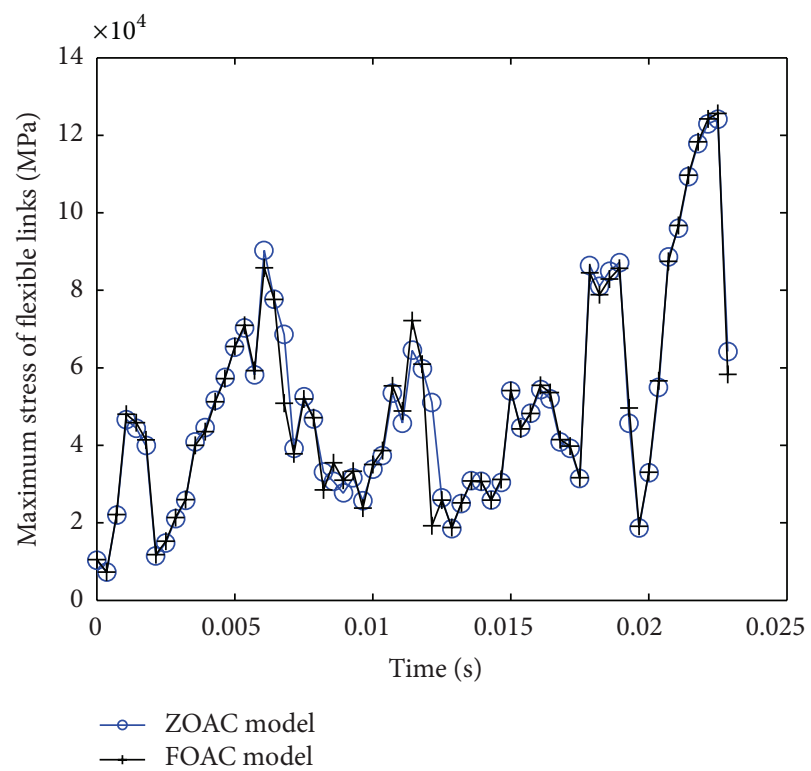

(d)

FIGURE 8: (a) and (b) are elastic displacements of the moving platform along with $X, Y$ direction, respectively, (c) is elastic rotational angle of the moving platform in $X Y$ planar, and (d) is maximum stress response of all flexible links through numerical calculation, when $w=$ $350 \pi \mathrm{rad} / \mathrm{s}$.

$10 \pi \mathrm{rad} / \mathrm{s}$ and $350 \pi \mathrm{rad} / \mathrm{s}$, respectively. Let the moving platform continuously operate four motion cycles along with the given circle trajectory Equation (32). Firstly, considering the parameter $w=10 \pi \mathrm{rad} / \mathrm{s}$ in (32), in this case, the acceleration of the moving platform is $4 \mathrm{~g}$ ( $\mathrm{g}$ is weight acceleration), and the maximum absolute angle speed of the drive joints $w_{i}$, $i=1,2,3$ reaches to $5.46,5.19$, and $5.2 \mathrm{rad} / \mathrm{s}$, respectively. Figure 4 shows elastic displacements and rotation angle of the moving platform with respect to four motion cycles based on the ZOAC model and the FOAC model when $w=$ $10 \pi \mathrm{rad} / \mathrm{s}$, respectively. Figure 5 shows elastic displacement responses which are located at the 1/3 length of active link 1 .
In all figures, the slide line with $\circ$ is a result using the ZOAC model, the slide line with + is a result using the FOAC model, and the slide line with $*$ is a result using ANSYS simulation. Figure 6(a) shows numerical result of the maximum stress response of flexible links; meanwhile, the simplified 3D model is developed by SolidWorks software and then imported into Workbench environment which is a simulation module of ANSYS 13.0 software for transient dynamic analysis as shown in Figure 6(b). All curves show periodical change in Figures 4-6.

Form Figures 4, 5, and 6(a), we can find that the numerical results are very close using the ZOAC model and the FOAC 


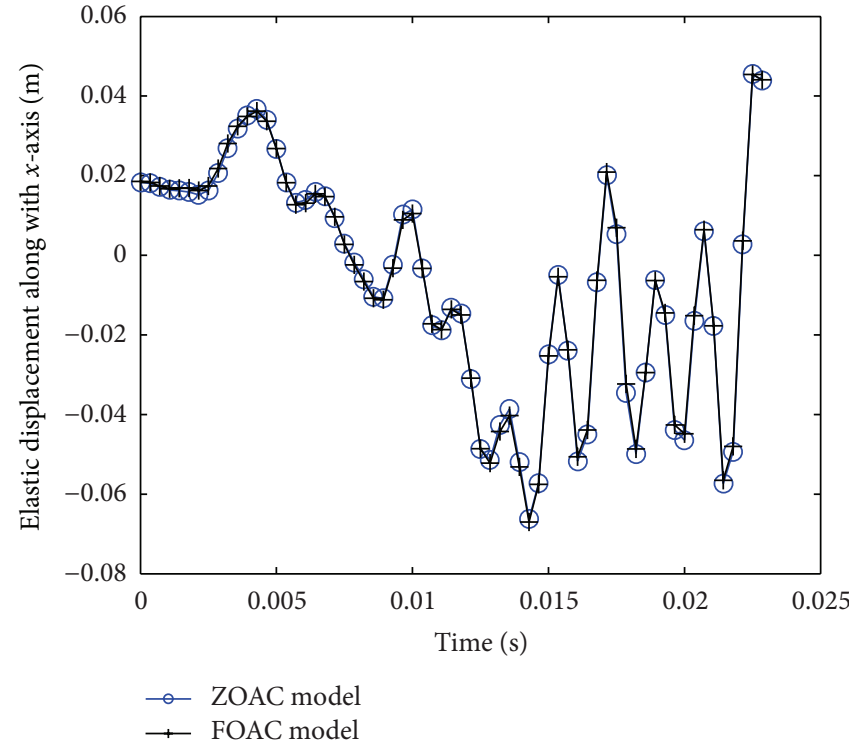

(a)



(b)

FIGURE 9: : (a) and (b) are elastic displacements of along with $X, Y$ direction which are located at the $1 / 3$ length of the active link 1 , respectively, when $w=350 \pi \mathrm{rad} / \mathrm{s}$.

model. The influence of dynamic stiffening on dynamic characteristic of the system can be neglected when $w=$ $10 \pi \mathrm{rad} / \mathrm{s}$. Reviewing Figures 6(a) and 6(b), one can find that numerical results are similar with ANSYS simulation results, and the change law of the curves are consistent and show periodic variation. Then, the validity of ZOAC model and FOAC model is verified.

To illustrate the validity of the theory model, modal analysis module of Workbench is used to calculate natural frequencies of the system. Theory results using the theory model and ANSYS result using ANSYS-Workbench software are listed in Table 2. From Table 2, we can find that the difference of the first natural frequency is $0.99 \%$ and very small, and the difference of the second natural frequency is $8.91 \%$, the difference of the third natural frequency is big and reaches to $37.14 \%$, the fourth-order is $11.74 \%$, meanwhile, all the first three-order natural frequencies are smaller than the fourth-order natural frequency using two methods. The existence of difference can be explained as follows: (1) all components are flexible in the ANSYS simulation, but, just only the links are flexible in theory model, (2) Meshing is different, and (3) solver may be different. Form the difference toleration and the consistent of the natural frequency change, ANSYS result can be used to verify the validity of the theory model.

To further verify, influence of stiffening dynamic of 3RRR flexible parallel robots is slight and can be neglected in the mathematics model. Let $w=350 \pi \mathrm{rad} / \mathrm{s}$ in (32). Then, the acceleration of the moving platform is $4836 \mathrm{~g}$ (Unbelievable), and the maximum absolute angle speed of the drive joints $w_{i}, i=1,2,3$ reaches to $191.02(30.04 \mathrm{~Hz})$,
TABLE 2: The first four-order natural frequencies of the system $(\mathrm{Hz})$.

\begin{tabular}{lcccc}
\hline & $w_{1}$ & $w_{2}$ & $w_{3}$ & $w_{4}$ \\
\hline Theory result & 27.177 & 38.146 & 65.794 & 307.4 \\
ANSYS result & 27.446 & 34.74 & 41.359 & 348.29 \\
Difference & $0.99 \%$ & $8.93 \%$ & $37.14 \%$ & $11.74 \%$ \\
\hline
\end{tabular}

$181.77(28.93 \mathrm{~Hz})$, and $182.58 \mathrm{rad} / \mathrm{s}(29.06 \mathrm{~Hz})$ and exceeds the first natural frequency $27.177 \mathrm{~Hz}$ (or $27.446 \mathrm{~Hz}$ ), as shown in Figures 8 and 9. Figures 8(a), 8(b), and 8(c) are elastic displacements and rotation angle of the moving platform with respect to four motion cycles using the ZOAC model and the FOAC model when $w=350 \pi \mathrm{rad} / \mathrm{s}$, respectively. Figure $8(\mathrm{~d})$ is the maximum stress response of flexible links with respect to four motion cycles. Figures 9 (a) and 9(b) are elastic displacement responses along with $X$ - axis and $Y$-axis which are located at the $1 / 3$ length of active link 1 , respectively.

From Figures 8 and 9, we find that although the acceleration of the moving platform has reached to $4836 \mathrm{~g} \mathrm{~m} / \mathrm{s}^{2}$ and the angle speed of the drive joints has exceeded the first natural frequency of the system, all curves using the ZOAC model are still very close to the relevant curves using the FOAC model. The influence of dynamic stiffening is still very slight. At the moment, elastic displacements and rotational angle of the moving platform and flexible link 1 increase two orders of magnitude more than $w=10 \pi \mathrm{rad} / \mathrm{s}$. The maximum stress of flexible links changes from $67.99 \mathrm{MPa}$ to $125600 \mathrm{MPa}$ and increases four orders of magnitude. When 
increasing $w$ in (32) again, there are just only increasing vibration amplitude of flexible links and the moving platform and maximum stress amplitude of flexible links; numerical results of the ZOAC model are still close to those of the FOAC model, and the difference between the ZOAC model and FOAC model is very slight.

From the above analysis, we can conclude that the influence of dynamic stiffening of planar 3-RRR flexible parallel robots is very slight and can fully be neglected for elastic dynamic modeling and the conventional ZOAC model can completely reveal dynamic characteristic of the system. The reasons can be explained as follows. (1) Reviewing $\mathbf{K}_{d}=\sum_{e} \mathbf{D}_{e}^{T} \mathbf{B}^{T}\left(g_{1 e} \widetilde{\mathbf{H}}_{e}+\mathbf{N}_{9}^{T} \ddot{\varphi}-\mathbf{N}_{8} \dot{\varphi}^{2}\right) \mathbf{B D}_{e}$, where $\ddot{\varphi}, g_{1 e}$ may take positive or negative value, the values of the first two terms may be positive or negative in bracket, and the third term is positive. Then, the value of the expression in brackets will be small and its sign is indeterminate. After summing with respect to all elements, $\mathbf{K}_{d}$ is much smaller than $\mathbf{K}_{1}$, so that $\mathbf{K}_{d}$ can be neglected in (31). (2) Because of the coupling among flexible links, the influence of dynamic stiffening which is related to rigid-body motion will be weakened.

\section{Conclusions}

The FOAC model of planar 3-RRR flexible parallel robots with six flexible links was established through the finite element method and the Lagrange equation, in which dynamic stiffening term was considered. The explicit expression of dynamic stiffening was presented. The constraint equations of the system that include the rigid body motion constraints, elastic deformation motion constraints, and dynamics constraints of the moving platform were studied in detail. For comparing the FOAC model with the conventional ZOAC model, numerical simulation was given under two different speeds of the moving platform. The results indicated that the conventional ZOAC model can fully reveal dynamic characteristic of the system and that the influence of dynamic stiffening is very slight and can be neglected for dynamic modeling of planar 3-RRR flexible parallel robots. So, the modeling process can be simplified. Meanwhile, the commercial software ANSYS 13.0 is used to confirm the validity of the zero-order approximation coupling model.

\section{Appendix}

\section{Conflict of Interests}

The authors declared that they have no conflicts of interest to this work.

\section{Acknowledgments}

This research was supported by the National Natural Science Foundation of China (Grant no. 91223201), the Natural Science Foundation of Guangdong Province (Grant no. S2013030013355), Project GDUPS (2010), and the Fundamental Research Funds for the Central Universities (2012ZP0004). This support is greatly acknowledged.

\section{References}

[1] M. Benosman and G. 1. Vey, "Control of flexible manipulators: a survey," Robotica, vol. 22, no. 5, pp. 533-545, 2004.

[2] S. K. Dwivedy and P. Eberhard, "Dynamic analysis of flexible manipulators, a literature review," Mechanism and Machine Theory, vol. 41, no. 7, pp. 749-777, 2006. 
[3] A. A. Shabana, "Flexible multibody dynamics: review of past and recent developments," Multibody System Dynamics, vol. 1, no. 2, pp. 189-222, 1997.

[4] A. G. Erdman and G. N. Sandor, "Kineto-elastodynamicsa review of the state of the art and trends," Mechanism and Machine Theory, vol. 7, no. 1, pp. 19-33, 1972.

[5] X. M. Zhang, C. J. Shao, and A. G. Erdman, "Active vibration controller design and comparison study of flexible linkage mechanism systems," Mechanism and Machine Theory, vol. 37, no. 9, pp. 985-997, 2002.

[6] X. M. Zhang, C. J. Shao, Y. W. Shen, and A. G. Erdman, "Complex mode dynamic analysis of flexible mechanism systems with piezoelectric sensors and actuators," Multibody System Dynamics, vol. 8, no. 1, pp. 51-70, 2002.

[7] J. D. Lee and Z. Geng, "A dynamic model of a flexible stewart platform," Computers \& Structures, vol. 48, no. 3, pp. 367-374, 1993.

[8] X. Y. Wang and J. K. Mills, "FEM dynamic model for active vibration control of flexible linkages and its application to a planar parallel manipulator," Applied Acoustics, vol. 66, no. 10, pp. 1151-1161, 2005.

[9] X. Y. Wang and J. K. Mills, "Dynamic modeling of a flexiblelink planar parallel platform using a substructuring approach," Mechanism and Machine Theory, vol. 41, no. 6, pp. 671-687, 2006.

[10] X. P. Zhang, J. K. Mills, and W. L. Cleghorn, "Experimental implementation on vibration mode control of a moving 3-PRR flexible parallel manipulator with multiple PZT transducers," Journal of Vibration and Control, vol. 16, no. 13, pp. 2035-2054, 2010.

[11] Q. H. Zhang, X. M. Zhang, and J. L. Liang, "Dynamic analysis of planar 3-RRR flexible parallel robot," in Proceedings of the IEEE International Conference on Robotics and Biomimetics (ROBIO '12), pp. 154-159, Guangzhou, China, December 2012.

[12] Q. H. Zhang and X. M. Zhang, "Dynamic modeling and analysis of planar 3-RRR flexible parallel robots," Journal of Vibration Engineering, vol. 26, no. 2, pp. 239-245, 2013.

[13] Q. H. Zhang and X. M. Zhang, "Dynamic analysis of planar 3-RRR flexible parallel robots under uniform temperature change," Journal of Vibration and Control, 2013.

[14] G. P. Cai, J. Z. Hong, and S. X. Yang, "Model study and active control of a rotating flexible cantilever beam," International Journal of Mechanical Sciences, vol. 46, no. 6, pp. 871-889, 2004.

[15] T. R. Kane, R. R. Ryan, and A. K. Banerjee, "Dynamics of a cantilever beam attached to a moving base," Journal of Guidance, Control, and Dynamics, vol. 10, no. 2, pp. 139-151, 1987.

[16] J. M. Mayo, D. García-Vallejo, and J. Domínguez, "Study of the geometric stiffening effect: comparison of different formulations," Multibody System Dynamics, vol. 11, no. 4, pp. 321-341, 2004.

[17] U. Lugrís, M. A. Naya, J. A. Pérez, and J. Cuadrado, "Implementation and efficiency of two geometric stiffening approaches," Multibody System Dynamics, vol. 20, no. 2, pp. 147-161, 2008.

[18] D. J. Zhang, Y. W. Liu, and R. L. Huston, "On dynamic stiffening of flexible bodies having high angular velocity," Mechanics of Structures and Machines, vol. 24, no. 3, pp. 313-329, 1996.

[19] Q. Yu and J. Z. Hong, "Some topics on flexible multibody system dynamics," Advances in Mechanics, vol. 29, no. 2, pp. 145-155, 1999.

[20] S. C. Wu and E. J. Haug, "Geometric non-linear substructure for dynamics of flexible mechanical systems," International Journal for Numerical Methods in Engineering, vol. 26, no. 10, pp. 22112226, 1988.

[21] H. Yang, Study of dynamic modeling theory and experiments for rigid-flexible coupling systems [Ph.D. thesis], Shanghai Jiaotong University, Shanghai, China, 2002. (Chinese).

[22] J. Y. Liu and H. Lu, "Rigid-flexible coupling dynamics of threedimensional hub-beams system," Multibody System Dynamics, vol. 18, no. 4, pp. 487-510, 2007. 

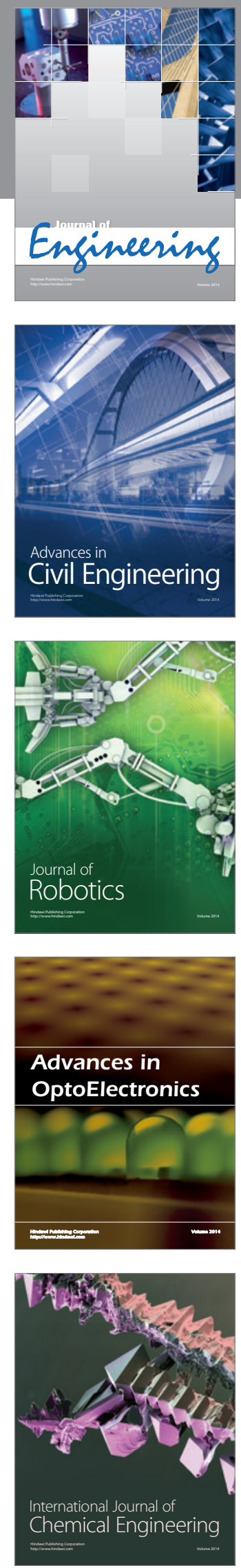

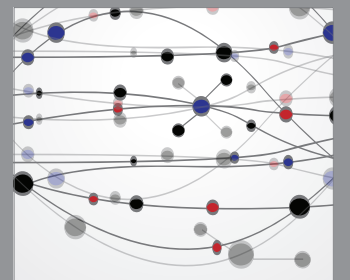

The Scientific World Journal
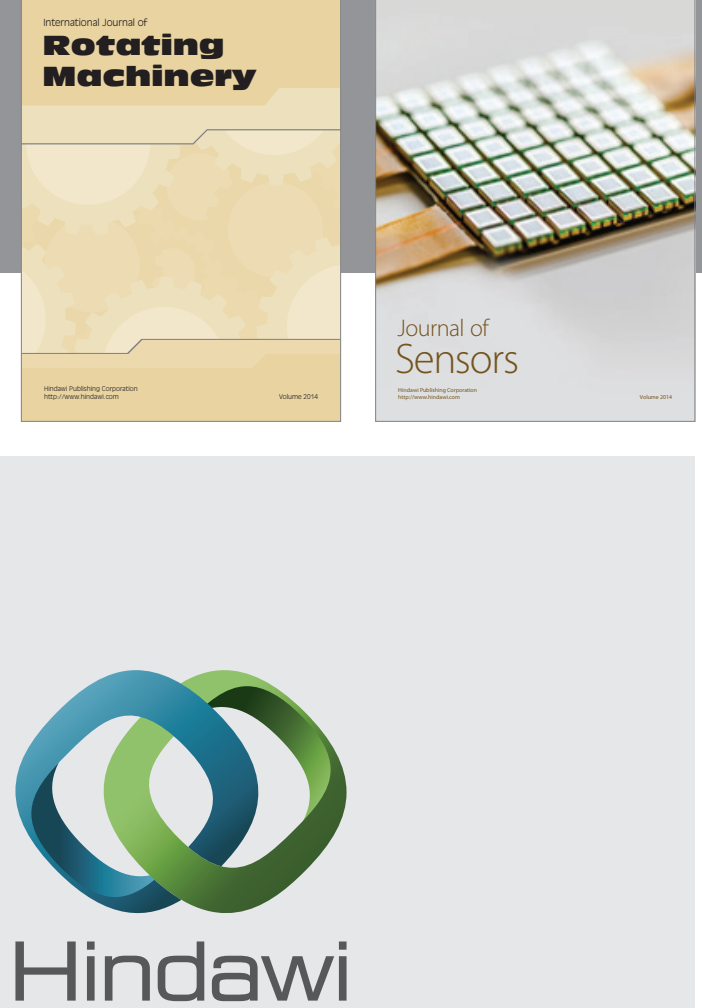

Submit your manuscripts at http://www.hindawi.com
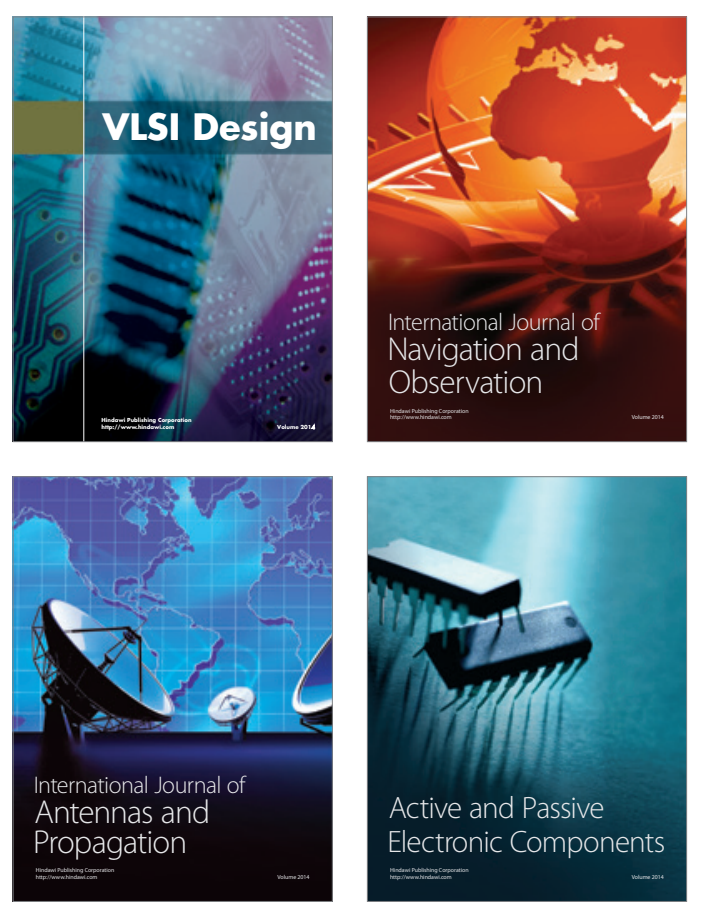
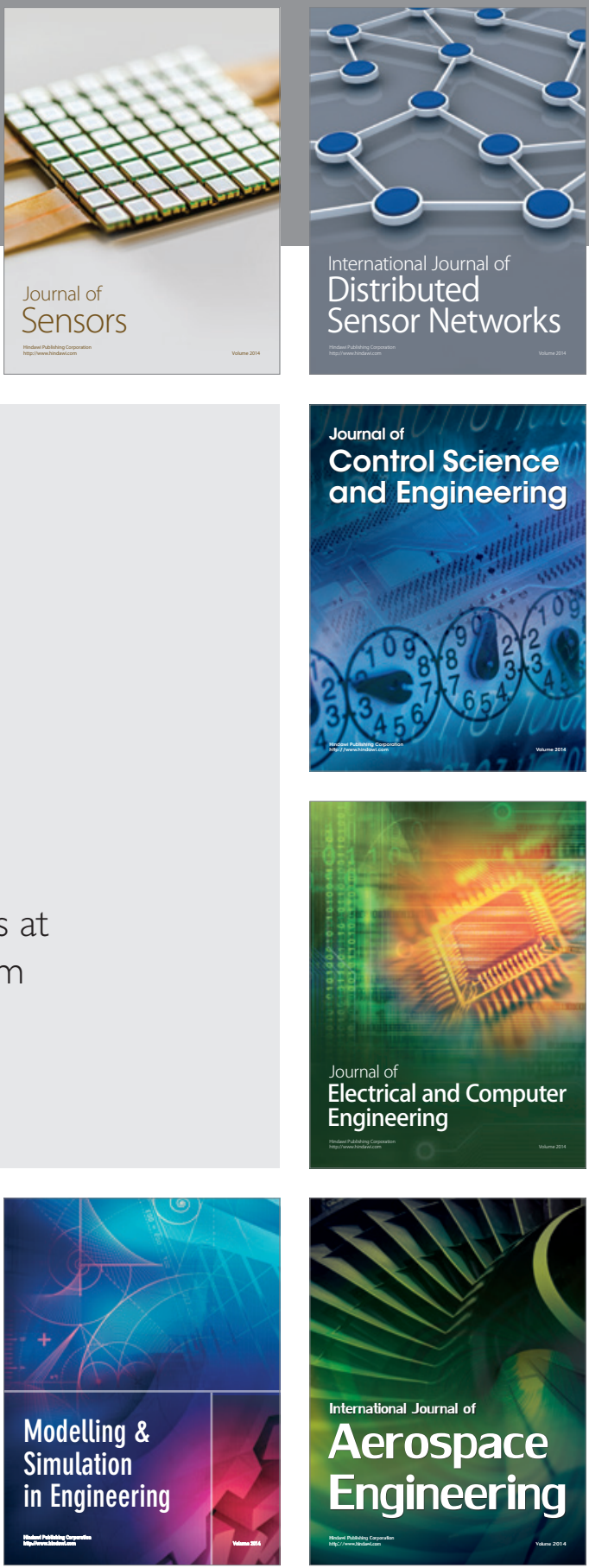

Journal of

Control Science

and Engineering
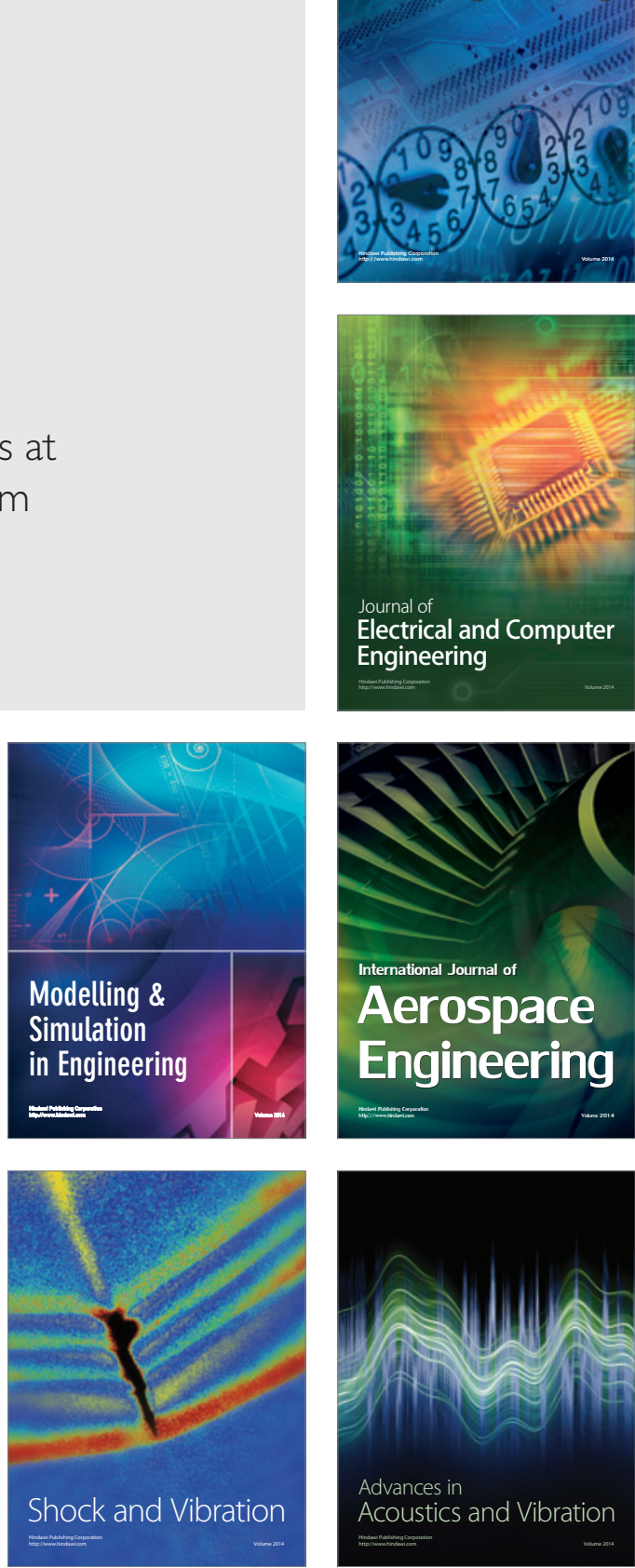